YITP/K-1120

q-alg/9508013

\title{
QUANTUM LIE ALGEBRAS ASSOCIATED TO $U_{q}\left(g l_{n}\right)$ AND $U_{q}\left(s l_{n}\right)$
}

\author{
Gustav W. Delius 网 and Andreas Hüffmann \\ Department of Mathematics, King's College London \\ Strand, London WC2R 2LS, Great Britain \\ e-mail: delius@mth.kcl.ac.uk and aha@mth.kcl.ac.uk \\ Mark D. Gould \\ Department of Mathematics, University of Queensland \\ Brisbane Qld 4072, Australia. \\ Yao-Zhong Zhang \\ Yukawa Institute for Theoretical Physics \\ Kyoto University, Kyoto 606, Japan \\ e-mail: yzzhang@yukawa.kyoto-u.ac.jp
}

\begin{abstract}
Quantum Lie algebras $\mathcal{L}_{q}(g)$ are non-associative algebras which are embedded into the quantized enveloping algebras $U_{q}(g)$ of Drinfeld and Jimbo in the same way as ordinary Lie algebras are embedded into their enveloping algebras. The quantum Lie product on $\mathcal{L}_{q}(g)$ is induced by the quantum adjoint action of $U_{q}(g)$. We construct the quantum Lie algebras associated to $U_{q}\left(g l_{n}\right)$ and $U_{q}\left(s l_{n}\right)$. We determine the structure constants and the quantum root systems, which are now functions of the quantum parameter $q$. They exhibit an interesting duality symmetry under $q \leftrightarrow 1 / q$.
\end{abstract}

${ }^{*}$ On leave from Department of Physics, Bielefeld University, Germany 
1. The theory of classically integrable systems relies heavily on Lie algebras and root systems. The discovery [1, 2] of the quantum deformations $U_{q}(g)$ of the universal enveloping algebras $U(g)$ of Lie algebras $g$ has led to major advances in the theory of quantum integrable systems. Many constructions in the theory of classically integrable systems do however require the use of Lie algebras rather than their enveloping algebras. An example of this are the values of the conserved charges on the solitons in affine Toda theory [3].

To generalize these constructions to the quantum level one would like to have the concept of a quantum Lie algebra $\mathcal{L}_{q}(g)$ which is related to the quantized enveloping algebra $U_{q}(g)$ in the same manner as a Lie algebra is related to its enveloping algebra. Such objects were introduced in 团 and will be reviewed below. In this paper we construct quantum Lie algebras associated to $g l_{n}$ and $s l_{n}$.

2. The quantized enveloping algebra $U_{q}\left(s l_{n}\right)$ is the unital associative algebra over $\mathbb{C}((t))$, the field of fractions for the ring of formal power series in the indeterminate $t$, with generators $x_{i}^{ \pm}, h_{i},(i=1, \cdots, n-1)$ and relations

$$
\begin{aligned}
& {\left[h_{i}, h_{j}\right]=0, \quad\left[h_{i}, x_{j}^{ \pm}\right]= \pm a_{i j} x_{j}^{ \pm}, \quad\left[x_{i}^{+}, x_{j}^{-}\right]=\delta_{i j} \frac{q^{h_{i}}-q^{-h_{i}}}{q-q^{-1}},} \\
& x_{i}^{ \pm} x_{i}^{ \pm} x_{j}^{ \pm}-\left(q+q^{-1}\right) x_{i}^{ \pm} x_{j}^{ \pm} x_{i}^{ \pm}+x_{j}^{ \pm} x_{i}^{ \pm} x_{i}^{ \pm}=0 \quad(|i-j|=1), \\
& x_{i}^{ \pm} x_{j}^{ \pm}=x_{j}^{ \pm} x_{i}^{ \pm} \quad(|i-j| \geq 2) .
\end{aligned}
$$

where we have defined $q=e^{t}$. Here $\left(a_{i j}\right)$ denotes the Cartan matrix of type $A_{n-1}$, i.e., $a_{i i}=2, a_{i j}=-1(|i-j|=1),=0$ (otherwise). We define $U_{q}\left(g l_{n}\right)$ by adjoining to $U_{q}\left(s l_{n}\right)$ an element $h_{n}$ which belongs to the center. The Hopf algebra structure is given by the coproduct $\Delta$, the antipode $S$ and the counit $\epsilon$

$$
\begin{aligned}
& \Delta\left(h_{i}\right)=h_{i} \otimes 1+1 \otimes h_{i}, \quad \Delta\left(x_{i}^{ \pm}\right)=x_{i}^{ \pm} \otimes q^{-h_{i} / 2}+q^{h_{i} / 2} \otimes x_{i}^{ \pm}, \\
& S\left(h_{i}\right)=-h_{i}, \quad S\left(x_{i}^{ \pm}\right)=-q^{\mp 1} x_{i}^{ \pm}, \quad \epsilon\left(h_{i}\right)=\epsilon\left(x_{i}^{ \pm}\right)=0 .
\end{aligned}
$$

Note that our conventions here differ from those of [4] by $q \leftrightarrow q^{-1}$ in order to conform to [5, 7, 8]. The adjoint action of $U_{q}(g)$ on itself is given by, using Sweedler's notation [间],

$$
x \circ y=\sum x_{(1)} y S\left(x_{(2)}\right), \quad x, y \in U_{q}(g) .
$$

The Cartan involution $\theta$ is the algebra automorphism defined by

$$
\theta\left(x_{i}^{ \pm}\right)=x_{i}^{\mp}, \quad \theta\left(h_{i}\right)=-h_{i} .
$$

It is a coalgebra antiautomorphism, i.e., $\Delta \cdot \theta=(\theta \otimes \theta) \cdot \Delta^{T}$ and $\epsilon \cdot \theta=\theta \cdot \epsilon$, and it satisfies $S \cdot \theta=\theta \cdot S^{-1}$. There is also an involutive algebra antiautomorphism $\dagger: a \mapsto a^{\dagger}$ defined by

$$
\left(x_{i}^{ \pm}\right)^{\dagger}=x_{i}^{\mp}, \quad\left(h_{i}\right)^{\dagger}=h_{i}
$$

which is a coalgebra automorphism and satisfies $S \cdot \dagger=\dagger \cdot S^{-1}$. The diagram automorphism $\tau$, defined by

$$
\tau\left(x_{i}^{ \pm}\right)=-x_{n-i}^{ \pm}, \quad \tau\left(h_{i}\right)=h_{n-i}(i \leq n-1), \quad \tau\left(h_{n}\right)=-h_{n},
$$


extends to a Hopf-algebra automorphism.

3. A central concept in the theory of quantum Lie algebras [4] is $q$-conjugation which in $\mathbb{C}((t))$ maps $t \mapsto-t$, i.e. $q \mapsto q^{-1}$.

Definition 1 a) q-conjugation $\sim: \mathbb{C}((t)) \rightarrow \mathbb{C}((t)), a \mapsto \tilde{a}$ is the field automorphism defined by $\tilde{t}=-t$.

b) Let $M, N$ be $\mathbb{C}((t))$-modules. A map $\phi: M \rightarrow N$ is q-linear if $\phi(\lambda a)=$ $\tilde{\lambda} \phi(a), \forall a \in M, \lambda \in \mathbb{C}((t))$.

c) Let $A, B$ be algebras over $\mathbb{C}((t))$. A q-linear map $\phi: A \rightarrow B$ is an algebra q-homomorphism if it respects the algebra product, i.e., if $\forall a, a^{\prime} \in A, \phi\left(a \overline{\left.a^{\prime}\right)=\phi(a) \phi\left(a^{\prime}\right)}\right.$. q-anti-isomorphims, q-automorphisms, etc., are defined analogously.

Note the analogy between the concepts of $q$-conjugation and complex conjugation and between $q$-linear maps and anti-linear maps.

Definition 2 q-conjugation on $U_{q}(g)$ is the algebra q-automorphism $\sim: U_{q}(g) \rightarrow U_{q}(g)$ that extends q-conjugation on $\mathbb{C}((t))$ by acting as the identity on the generators $x_{i}^{ \pm}$ and $h_{i}$.

This definition is consistent because the relations (11) are invariant uder $q \mapsto q^{-1}$. $q$-conjugation is a coalgebra q-antiautomorphism of $U_{q}(g)$, i.e., $\epsilon \cdot \sim=\sim \cdot \epsilon, \Delta \cdot \sim=\sim$ $\cdot \Delta^{T}$ and it satisfies $S \cdot \sim=\sim \cdot S^{-1}$. We define a tilded Cartan involution and a tilded antipode as compositions

$$
\tilde{S}=\sim \cdot S, \quad \tilde{\theta}=\sim \cdot \theta
$$

These behave well with respect to the adjoint action:

$$
\tilde{\theta}(a) \circ \tilde{\theta}(b)=\tilde{\theta}(a \circ b), \quad \tilde{S}(a) \circ \tilde{S}(b)=\tilde{S}\left(S^{-1}(a) \circ b\right), \quad \forall a, b \in U_{q}(g) .
$$

4. A Lie algebra $g$ is naturally embedded into its universal enveloping algebra $U(g)$. It forms a subspace of the enveloping algebra which under the adjoint action transforms in the adjoint representation. The Lie bracket on $g$ is given by the restriction of the adjoint action of $U(\mathrm{~g})$. This idea is extended to the quantum case by the following definition, introduced in [4] (and slightly modified to incorporate also the non-simple Lie algebra $g l_{n}$ ):

Definition 3 A quantum Lie algebra $\mathcal{L}_{q}(g)$ associated to a finite dimensional complex Lie algebra $g$ is a $\circ$ - submodule of $U_{q}(g)$, endowed with the quantum Lie bracket $[a \circ \beta]=a \circ b$, and which has the properties:

1. $\mathcal{L}_{q}(g)$ has the same dimension as $g$,

2. $\mathcal{L}_{q}(g)$ is a deformation of $g$, i.e., the isomorphism $U_{q}(g) / t U_{q}(g) \cong U(g)$ takes $\mathcal{L}_{q}(g) / t \mathcal{L}_{q}(g)$ isomorphically onto $g \subset U(g)$,

3. $\mathcal{L}_{q}(g)$ is invariant under $\tilde{\theta}, \tilde{S}$ and $\tau$. 
Remark: It was shown in [4] that if at least one o-module satisfying the first two properties exists, then there exist infinitely many and out of these one can always choose at least one statisfying also the last property. The last property plays a crucial role in the investigations into the general structure of quantum Lie algebras.

5. We will now show how to construct a quantum Lie algebra $\mathcal{L}_{q}\left(g l_{n}\right)$ starting from an expression for the universal R-matrix of $U_{q}\left(g l_{n}\right)$. Because $g l_{n}$ is not simple the universal R-matrix is not unique and therefore also the expressions for the quantum Lie algebra generators which we obtain will not be unique. Our construction is based on the results of [7, 8]. We introduce the elements $E_{i j} \in U_{q}\left(g l_{n}\right)$ defined recursively by

$$
\begin{aligned}
& E_{i j}=E_{i k} E_{k j}-q E_{k j} E_{i k} \quad(i<k<j \text { or } i>k>j), \quad E_{i, i \pm 1}=x_{i}^{ \pm}, \\
& E_{i i}-E_{i+1, i+1}=h_{i} \quad(i<n), \quad \sum_{i=1}^{n} E_{i i}=h_{n} .
\end{aligned}
$$

Let $\pi$ denote the vector representation of $g l_{n}$. Then $\pi\left(E_{i j}\right)=e_{i j} \forall i, j$, where $e_{i j}$ denotes the matrix $\left(\delta_{i a} \delta_{j b}\right)_{1 \leq a, b \leq n}$. We follow the convention of [7, 8] and define the dual representation $\pi^{*}$ not with the antipode, as would be standard, but as $\pi^{*}(a):=$ $\pi^{t}(\gamma(a))$ where $\gamma$ is the antiautomorphism defined by $\gamma\left(x_{i}^{ \pm}\right)=-x_{i}^{ \pm}, \gamma\left(h_{i}\right)=-h_{i}$. Rewriting a result by Jimbo [5] one finds that there exists a universal R-matrix $\mathcal{R}$ for $U_{q}\left(g l_{n}\right)$ such that

$$
\left(\pi_{i j}^{*} \otimes 1\right)(\mathcal{R})=\delta_{i \leq j} q^{i-j} \hat{E}_{i j}, \quad\left(\pi_{j i}^{*} \otimes 1\right)\left(\mathcal{R}^{T}\right)=\delta_{i \leq j} q^{i-j} \hat{E}_{j i},
$$

where we have defined

$$
\hat{E}_{i j}= \begin{cases}-\left(q-q^{-1}\right) q^{-\left(E_{i i}+E_{j j}-1\right) / 2} E_{i j} & i \neq j \\ q^{-E_{i i}} & i=j .\end{cases}
$$

(We use a generalized Kronecker delta notation, e.g., $\delta_{i \leq j}=1$ if $i \leq j, 0$ otherwise.) It is shown in [7, \& that the elements $T_{i j} \in U_{q}\left(g l_{n}\right)$ defined by

$$
\begin{aligned}
T_{i j} & =q^{i}\left(q-q^{-1}\right)^{-1}\left(\delta_{i j}-\left(\pi_{i j}^{*} \otimes 1\right)\left(\mathcal{R}^{T} \mathcal{R}\right)\right) \\
& =q^{i}\left(q-q^{-1}\right)^{-1}\left(\delta_{i j}-q^{-i-j} \sum_{k \leq \min (i, j)} q^{2 k} \hat{E}_{i k} \hat{E}_{k j}\right),
\end{aligned}
$$

transform as the components of a $\pi \otimes \pi^{*}$ tensor operator, i.e., that

$$
a \circ T_{i j}=T_{k l}\left(\pi_{k i} \otimes \pi_{l j}^{*}\right) \Delta(a), \quad \forall a \in U_{q}\left(g l_{n}\right) .
$$

The representation $\pi \otimes \pi^{*}$ is isomorphic to the adjoint representation of $U_{q}\left(g l_{n}\right)$. Classically the $T_{i j}$ go over into the $g l_{n}$ generators $\left.E_{i j}\right|_{q=1}$. Thus the $T_{i j}$ span a o-module satisfying all requirements of definition 3 for $\mathcal{L}_{q}\left(g l_{n}\right)$ except property 3 .

This o-module also appears as the dual space to the space of left-invariant oneforms in the framework of the bicovariant differential calculus on quantum groups (see e.g. [g]) and it is a special case of the braided matrix Lie algebras of Majid [10]. 
6. The adjoint representation of $g l(n)$ is not irreducible. Correspondingly the omodule spanned by the $T_{i j}$ decomposes into a 1-dimensional module spanned by a Casimir $\mathcal{C}$ and a $\left(n^{2}-1\right)$-dimensional module spanned by elements $\mathcal{T}_{i j}$, where

$$
\mathcal{C}=\sum_{i=1}^{n} \frac{1-q^{-2}}{q^{2 n}-1} q^{i} T_{i i}, \quad \mathcal{T}_{i j}=T_{i j}-\delta_{i j} q^{i} \mathcal{C}
$$

7. A second o-module is spanned by the elements $\tau\left(T_{i j}\right)$. They transform as follows:

$$
\begin{aligned}
a \circ \tau\left(T_{i j}\right) & =\tau\left(\tau(a) \circ T_{i j}\right)=\tau\left(T_{k l}\right)\left(\pi_{k i} \otimes \pi_{l j}^{*}\right) \Delta(\tau(a)) \\
& =\tau\left(T_{k l}\right)\left(\pi_{\bar{k} \bar{\imath}}^{*} \otimes \pi_{\bar{l} \bar{\jmath}}\right) \Delta(a) .
\end{aligned}
$$

(Repeated indices are summed over) We have introduced the notation $\bar{\imath}=n+1-i$ and have used that $\Delta(\tau(a))=(\tau \otimes \tau) \Delta(a)$ and $\pi_{i j}^{*}(a)=\pi_{\bar{\imath} \bar{\jmath}}(\tau(a))$. As a new basis in this second module we choose

$$
V_{i j}:=-\tau\left(T_{\bar{k} \bar{l}}\right)\left(R_{\pi \pi^{*}}\right)_{l k i j}
$$

where

$$
\begin{aligned}
\left(R_{\pi \pi^{*}}\right)_{i j k l} & :=\left(\pi_{i k} \otimes \pi_{j l}^{*}\right) \mathcal{R} \\
& =\delta_{i k} \delta_{j l}+\delta_{i j} \delta_{k l}\left(\left(q^{-1}-1\right) \delta_{i k}-\left(q-q^{-1}\right) q^{k-i} \delta_{i>k}\right) .
\end{aligned}
$$

The $V_{i j}$ transform in the same way as the $T_{i j}$ :

$$
\begin{aligned}
a \circ V_{i j} & =V_{k l}\left(R_{\pi \pi^{*}}^{-1}\right)_{k l s r}\left(\left(\pi_{r p}^{*} \otimes \pi_{s q}\right) \Delta(a)\right)\left(R_{\pi \pi^{*}}\right)_{q p i j} \\
& =V_{k l}\left(\pi_{k i} \otimes \pi_{l j}^{*}\right) \Delta(a),
\end{aligned}
$$

where we have used the intertwining property of the R-matrix. They also have the same classical limit. Again we decompose into the $\left(n^{2}-1\right)$-dimensional and the trivial module generated by $\mathcal{V}_{i j}$ and $\mathcal{B}$ respectively, where

$$
\mathcal{B}=\sum_{i=1}^{n} \frac{1-q^{-2}}{q^{2 n}-1} q^{i} V_{i i}=-q^{-n} \tau(\mathcal{C}), \quad \mathcal{V}_{i j}=V_{i j}-\delta_{i j} q^{i} \mathcal{B}
$$

8. Because the $T_{i j}$ and the $V_{i j}$ have the same transformation properties, their sums $1 / 2\left(T_{i j}+V_{i j}\right)$ also generate a o-module. This module contains an $\left(n^{2}-1\right)$-dimensional submodule generated by $\mathcal{X}_{i j}:=\frac{1}{2}\left(\mathcal{T}_{i j}+\mathcal{V}_{i j}\right)$ which is $\tau$-invariant:

$$
\begin{aligned}
\tau\left(\mathcal{X}_{i j}\right) & =\frac{1}{2}\left(\tau\left(\mathcal{T}_{i j}\right)-\mathcal{T}_{\bar{k} \bar{l}}\left(R_{\pi \pi^{*}}\right)_{l k i j}\right) \\
& =-\mathcal{X}_{\bar{k} \bar{l}}\left(R_{\pi \pi^{*}}\right)_{l k i j}-\frac{1}{2} \tau\left(\mathcal{T}_{\bar{r} \bar{s}}\right)\left(R_{\pi \pi^{*}}\right)_{s r \bar{k} \bar{l}}\left(R_{\pi \pi^{*}}\right)_{l k i j}+\frac{1}{2} \tau\left(\mathcal{T}_{i j}\right) \\
& =-\mathcal{X}_{\bar{k} \bar{l}}\left(R_{\pi \pi^{*}}\right)_{l k i j} .
\end{aligned}
$$

For the last equality we used that $\left(R_{\pi \pi^{*}}\right)_{s r \bar{k} \bar{l}}\left(R_{\pi \pi^{*}}\right)_{l k i j}=\delta_{\bar{r} i} \delta_{\bar{s} j}+\left(q^{-2 n}-1\right) P_{\bar{r} \bar{s} i j}$, where $P$ is the projector onto the 1-dimensional orbit, and that $\mathcal{T}$, which lies in the 
$\left(n^{2}-1\right)$-dimensional module, vanishes when contracted with $P$. As basis vectors we choose

$$
\begin{aligned}
X_{i j} & =q^{(j-i-1) / 2} \mathcal{X}_{i j} \quad(i \neq j=1, \cdots n) \\
H_{i} & =\mathcal{X}_{i i}-q^{-1} \mathcal{X}_{i+1, i+1} \quad(i=1, \ldots, n-1)
\end{aligned}
$$

They satisfy

$$
\begin{aligned}
& \tilde{\theta}\left(X_{i j}\right)=(-1)^{i+j+1} X_{j i}, \quad \tilde{\theta}\left(H_{i}\right)=-H_{i}, \\
& \tilde{S}\left(X_{i j}\right)=-q^{j-i} X_{i j}, \quad \tilde{S}\left(H_{i}\right)=-H_{i}, \\
& \left(X_{i j}\right)^{\dagger}=X_{j i}, \quad\left(H_{i}\right)^{\dagger}=H_{i} .
\end{aligned}
$$

The $\tau$-invariant 1-dimensional module is generated by the Casimir $K=\mathcal{C}+q^{n} \mathcal{B}$, which satisfies $\tau(K)=\tilde{\theta}(K)=\tilde{S}(K)=-K, K^{\dagger}=K$. Note that the Casimir $\mathcal{C}+\mathcal{B}$ is not $\tau$ invariant.

Thus we have obtained the quantum Lie algebra $\mathcal{L}_{q}\left(g l_{n}\right)$ spanned by a basis $\left\{X_{i j} \mid i, j=1, \cdots, n\right\} \cup\left\{H_{i} \mid i=1, \cdots, n-1\right\} \cup\{K\}$ which satisfies all the conditions of definition 3 .

9. To calculate the structure constants of $\mathcal{L}_{q}\left(g l_{n}\right)$ we use the formulas

$$
\begin{aligned}
T_{i j} \circ \hat{T}_{k l} & =\left(\pi_{r k} \otimes \pi_{s l}^{*}\right) \Delta\left(T_{i j}\right) \\
& =\frac{q^{i}}{q-q^{-1}}\left(\delta_{i j} \delta_{r k} \delta_{s l}-\left(R_{\pi \pi^{*}}\right)_{r i f a}\left(R_{\pi^{*} \pi}\right)_{c f j k}\left(R_{\pi^{*} \pi^{*}}\right)_{s a h b}\left(R_{\pi^{*} \pi^{*}}\right)_{b h c l}\right), \\
\tau\left(T_{\bar{\jmath}}\right) \circ \hat{T}_{k l} & =\left(\pi_{r k} \otimes \pi_{s l}^{*}\right) \Delta\left(\tau\left(T_{\bar{\jmath}}\right)\right)=\left(\pi_{\bar{r} \bar{k}}^{*} \otimes \pi_{\bar{s} \bar{l}}\right) \Delta\left(T_{\bar{\jmath}}\right) \\
& =\frac{q^{n+1-j}}{q-q^{-1}}\left(\delta_{i j} \delta_{r k} \delta_{s l}-\left(R_{\pi^{*} \pi^{*}}\right)_{\bar{r} \bar{\jmath} f a}\left(R_{\pi^{*} \pi^{*}}\right)_{c f \bar{\imath} \bar{k}}\left(R_{\pi \pi^{*}}\right)_{\bar{s} a h b}\left(R_{\pi^{*} \pi}\right)_{b h c \bar{l}}\right)
\end{aligned}
$$

which follow from $\Delta\left(R^{T} R\right)=R_{12}^{T} R_{13}^{T} R_{13} R_{12}$. We also need the relation

$$
X_{i i}=-\sum_{k=1}^{n-1} q^{i-k} \frac{q^{2 k}-1}{q^{2 n}-1} H_{k}+\sum_{k=i}^{n-1} q^{i-k} H_{k}+q^{i} K .
$$

Tedious but straightforward calculations give

$$
\begin{aligned}
& {\left[H_{k} \circ X_{i j}\right]=l_{i j}\left(H_{k}\right) X_{i j}, \quad\left[X_{i j} \circ H_{k}\right]=-r_{i j}\left(H_{k}\right) X_{i j},} \\
& {\left[H_{i} \circ H_{j}\right]=f_{i j}{ }^{k} H_{k}, \quad\left[X_{i j} \circ X_{j i}\right]=g_{i j}{ }^{k} H_{k},} \\
& {\left[X_{i j} \circ X_{k l}\right]=\delta_{j k} \delta_{i \neq l} N_{i j l} X_{i l}+\delta_{i l} \delta_{j \neq k} M_{k i j} X_{k j},} \\
& {[K \circ a]=[a \circ K]=0 \quad \forall a \in \mathcal{L}_{q}\left(g l_{n}\right) .}
\end{aligned}
$$

where

$$
\begin{aligned}
l_{i j}\left(H_{k}\right) & =\frac{1}{2}\left(1+q^{n}\right)\left(q^{-k}\left(q \delta_{k i}-q^{-1} \delta_{k, i-1}\right)+q^{k-n}\left(q \delta_{k, j-1}-q^{-1} \delta_{k j}\right)\right), \\
r_{i j}\left(H_{k}\right) & =-l_{j i}\left(H_{k}\right), \\
f_{i j}{ }^{k} & =\delta_{i j} \frac{1}{2}\left(\delta_{k<i}\left(q+q^{-1}\right)\left(q^{k}-q^{-k}\right)+\delta_{k>i}\left(q+q^{-1}\right)\left(q^{n-k}-q^{-n+k}\right)\right.
\end{aligned}
$$




$$
\begin{gathered}
\left.+\delta_{k i}\left(q^{i+1}-q^{-i-1}+q^{n+1-i}-q^{-n-1+i}\right)\right) \\
+\delta_{i, j-1} \frac{1}{2}\left(\delta_{k \leq i}\left(q^{-k}-q^{k}\right)+\delta_{k>i}\left(q^{k-n}-q^{-k+n}\right)\right) \\
+\delta_{i, j+1} \frac{1}{2}\left(\delta_{k<i}\left(q^{-k}-q^{k}\right)+\delta_{k \geq i}\left(q^{k-n}-q^{-k+n}\right)\right), \\
g_{i j}^{k}=\frac{1}{2} q^{i-j}\left(\delta_{k<j}\left(q^{k}-q^{-k}\right)+\delta_{k \geq i}\left(q^{-k}+q^{-k+n}\right)-\delta_{k \geq j}\left(q^{-k}+q^{k-n}\right)\right), \\
N_{i j l}=\frac{1}{2} q^{-j+1 / 2}\left(1+q^{n}\right), \quad M_{k i j}=\tilde{N}_{k i j}
\end{gathered}
$$

One notices several properties:

1. For $t=0(q=1)$ these are the standard $g l_{n}$ Lie bracket relations.

2. The "quantum Cartan subalgebra" generators $H_{i}$ have non-vanishing quantum Lie brackets among themselves. However it is still commutative in the sense that $\left[H_{i} \circ H_{j}\right]=\left[H_{j} \circ H_{i}\right]$.

3. There are now two sets of roots, $L=\left\{l_{i j}\right\}$ and $R=\left\{r_{i j}\right\}$, related by (29). The combinations $a_{i j}=\left(l_{i j}+r_{i j}\right) / 2$ form the standard $g l_{n}$ root lattice, i.e., they satisfy $a_{i j}+a_{k l}=\delta_{j k} a_{i l}+\delta_{i l} a_{k j}$ and $a_{j i}=-a_{i j}$. This feature is probably true only for $g$ simply-laced. It is known that the lattice structure is broken in the non-simply laced cases of $g=C_{2}$ [四] and $g=G_{2}$ [11].

4. $\dagger$ is a quantum Lie algebra antiautomorphism, i.e.,

$$
\left[a^{\dagger} \circ b^{\dagger}\right]=[b \circ a]^{\dagger}, \quad \forall a, b \in \mathcal{L}_{q}(g) .
$$

5. The quantum Lie bracket is $q$-antisymmetric in the sense that

$$
\left[a^{q} \circ b^{q}\right]=-[b \circ a]^{q}, \quad \forall a, b \in \mathcal{L}_{q}(g),
$$

where we have defined the $q$-conjugation $a \mapsto a^{q}$ on $\mathcal{L}_{q}(g)$ as the $q$-linear map which extends the $q$-conjugation $\sim$ on $\mathbb{C}((t))$ to $\mathcal{L}_{q}(g)$ by acting as the identity on the basis elements $X_{i j}, H_{i}$ and $K$. (Note that this is not the same as the q-conjugation $\sim$ on $U_{q}(g)$ which does not leave $\mathcal{L}_{q}(g)$ invariant.) This $q$-antisymmetry of the quantum Lie bracket was observed also for $\mathcal{L}_{q}\left(s_{5}\right)$ [4] and $\mathcal{L}_{q}\left(G_{2}\right)$ [1].

6. The element $K$ decouples completely. Thus $\mathcal{L}_{q}\left(g l_{n}\right)$ is not simple.

7. We would like to stress that the relative simplicity of the structure constants arises only after symmetrizing the o-module with respect to the diagram automorphism $\tau$. The quantum adjoint action of the $T_{i j}$ on themselves is complicated and does not display any of the features mentioned in the above points 2 to 6 .

8. The structure constants display the symmetries $\left.l_{j i}\left(H_{k}\right)=-l_{i j} \widetilde{(H}_{k}\right), \quad r_{j i}\left(H_{k}\right)=$ $\left.-r_{i j} \widetilde{(H}_{k}\right)$ and $f_{i j}{ }^{k}=-\tilde{f}_{i j}{ }^{k}$, which were derived generally in [4] from the property (8) of $\tilde{\theta}$. 
9. For $n=3$ these Lie bracket relations reproduce those for $\mathcal{L}_{q}\left(s l_{3}\right)$ given in [4] (after replacing $q \leftrightarrow 1 / q$ and changing the normalization of the generators).

10. To obtain $\mathcal{L}_{q}\left(s l_{n}\right)$ inside $U_{q}\left(s l_{n}\right)$ one can repeat the above analyis, starting in paragraph 5 with the universal R-matrix of $U_{q}\left(s l_{n}\right)$ as given by Rosso [12]. Because the numerical R-matrices (R-matrices evaluated in representations) which we used for $g l_{n}$ are the same as those for $s l_{n}$, the formulation of the $\tau$-invariant $\left(n^{2}-1\right)$ dimensional o-module works as before and now gives $\mathcal{L}_{q}\left(s l_{n}\right)$. Also the structure constants for $\mathcal{L}_{q}\left(s l_{n}\right)$ are given by equations (27)-(32), simply dropping $K$.

11. We define the quantum Killing form $B$ on $\mathcal{L}_{q}\left(s l_{n}\right)$ by

$$
B(a, b)=-q^{-1} \operatorname{Tr}_{\pi}(\tilde{S}(a) b u),
$$

where $\operatorname{Tr}_{\pi}$ denotes the trace over the vector representation and $u$ is the element of $U_{q}\left(s l_{n}\right)$ expressing the square of the antipode as $S^{2}(a)=u a u^{-1}, \forall a \in U_{q}(g)$. This form is proportional to that defined in 4 . It has as its defining property the ad-invariance $B(a, c \circ b)=B(\tilde{S}(c) \circ a, b)$. It is $q$-linear in its first argument and linear in the second and satisfies $B(b, a)=\widetilde{B(a, b)}=B(\tilde{S}(a), S(\tilde{b}))=B(\tilde{\theta}(a), \tilde{\theta}(b))$. As explained in [4, it is not the restriction of Rosso's form [13] on $U_{q}(g)$ to $\mathcal{L}_{q}(g)$. On our basis the Killing form takes the values

$$
\begin{aligned}
& B\left(H_{i}, H_{k}\right)=\left(q+q^{-1}\right) \delta_{i k}-\delta_{i, k-1}-\delta_{i, k+1}, \\
& B\left(X_{i j}, X_{k l}\right)=\delta_{j k} \delta_{l i}, \quad B\left(H_{k}, X_{i j}\right)=0 .
\end{aligned}
$$

The ad-invariance of the Killing form leads to further relations among the structure constants derived in [4:

$$
g_{i j k}=q^{j-i} r_{i j}\left(H_{k}\right), \quad f_{i j k}=f_{i k j}, \quad N_{k i j}=-q^{j-i} N_{i j k} .
$$

Here we have lowered indices with the Killing form, e.g., $f_{i j k}=B\left(H_{k}, H_{l}\right) f_{i j}{ }^{l}$.

Acknowledgements G.W.D. thanks the Deutsche Forschungsgemeinschaft for a Habilitationsstipendium. A.H. thanks the EC for a research fellowship. Y.Z.Z. is financially supported by the Kyoto University Foundation.

\section{References}

[1] V.G. Drinfel'd, Hopf algebras and the quantum Yang-Baxter equation, Sov. Math. Dokl. 32 (1985) 254.

[2] M. Jimbo, A q-Difference Analogue of $U(g)$ and the Yang-Baxter Equation, Lett. Math. Phys. 10 (1985) 63.

[3] M. Freeman, Conserved charges and soliton solutions in affine Toda theory, Nucl. Phys. B433 (1995) 657.

[4] G.W. Delius, A. Hüffmann, On Quantum Lie Algebras and Quantum Root Systems, q-alg/9506017, submitted to Commun. Math. Phys. 
[5] M. Jimbo, A q-Analogue of $U(g l(N+1))$, Hecke Algebra, and the Yang-Baxter Equation, Lett. Math. Phys. 11 (1986) 247.

[6] M.E. Sweedler, Hopf algebras, Benjamin, New York (1969).

[7] J.R. Links, M.D. Gould, Casimir Invariants for Hopf Algebras, Rep. Math. Phys. 31 (1992) 91.

[8] M.D. Gould, J. Links, A.J. Bracken, Matrix elements and Wigner coefficients for $U_{q}\left(g l_{n}\right)$, J. Math. Phys. 33 (1992) 1008.

[9] P. Aschieri, L. Castellani, An introduction to noncommutative differential geometry on quantum groups, Int. J. Mod. Phys. A8 (1993) 1667.

[10] S. Majid, Quantum and Braided Lie Algebras, J. Geom. Phys. 13 (1994) 307. (1993).

[11] G.W. Delius, A. Hüffmann, unpublished.

[12] M. Rosso, An Analogue of P.B.W. Theorem and the Universal R-Matrix for $U_{h} \operatorname{sl}(N+1)$, Commun. Math. Phys. 124 (1989) 319.

[13] M. Rosso, Analogues de la forme de Killing et du theoreme d'Harish-Chandra pour les groupes quantiques, Ann. scient. Ec. Norm. Sup. 23 (1990) 445. 